\title{
Validación de los marcadores ArUco para el análisis de movimientos humanos
}

\author{
Jara, Nicolás ${ }^{a}$; Abad, José Luis ${ }^{b}$; Navarro, Joséc; Page, Álvaro ${ }^{\text {d* }}$ \\ ${ }^{a}$ ETSI Industriales, Universitat Politècnica de València (España), nijaget@etsii.upv.es, \\ ${ }^{b}$ ETSI Telecomunicación, Universitat Politècnica de València, joabos@ade.upv.es, \\ ${ }^{\mathrm{c}}$ Dpto. de Sistemas Informáticos y Computación, Universitat Politècnica de València, \\ jonaal1@doctor.upv.es, \\ ${ }^{\mathrm{d}}$ Instituto Universitario Mixto de Biomecánica, Universitat Politècnica de València, \\ afpage@ibv.upv.esc
}

\begin{abstract}
Although video-photogrammetry is the gold standard for human movement analysis, its complexity and high cost arouse interest in simpler and cheaper alternatives. One of them is videoanalysis, which allows analyzing movements in a plane. An alternative that allows the study of movements in $3 D$ is the uaugmented reality markers (AR markers), widely used in the field of robotics. These systems allow to analyze, in real time and with a single video camera, the position and orientation of objects with sufficient precision for use in many biomechanical applications. This article analyzes the accuracy of ArUco markers in the measurement of angles and displacements, comparing the movements measured with the system with two precision techniques: encoders of linear and angular displacements and a standar equipment of videophotogrammetry. The results show that markers can measure displacements with errors lower than those associated with human variability, so it would be possible to use this type of markers in a wide variety of biomechanical applications.
\end{abstract}

Keywords: Human movement, video analysis, OpenCV, ArUco, accuracy.

\section{Resumen}

Aunque la videofotogrametría es la técnica de referencia para el análisis de movimientos humanos, su complejidad y elevado coste hacen que se planteen otras alternativas más sencillas y baratas. Una de ellas es el videoanálisis, que permite analizar movimientos en un plano. Una alternativa que permite el estudio de movimientos $3 D$ es el uso de marcadores de realidad aumentada (AR), de amplio uso en el campo de la robótica. Estos sistemas permiten analizar, en tiempo real y con una única cámara de video, la posición y orientación de objetos con precisión suficiente para su uso en muchas aplicaciones biomecánicas. En este trabajo se analiza la precisión de los 
marcadores ArUco en la medida de ángulos y desplazamientos, comparando los movimientos medidos con el sistema con dos técnicas de precisión: encoders de desplazamientos lineales y giros, para las medidas estáticas, y un sistema de videofotogrametría de precisión, para las medidas en movimiento. Los resultados muestran que los marcadores pueden medir desplazamientos con errores inferiores a los asociados a la variabilidad humana, por lo que sería posible utilizar este tipo de marcadores para numerosas aplicaciones biomecánicas.

Palabras clave: Análisis de movimientos humanos, videoanálisis, marcadores de realidad virtual, ArUco, OpenCV, precisión.

\section{Introducción}

Aunque la videofotogrametría es la técnica de referencia para el análisis de movimientos humanos, su complejidad y elevado coste hacen que se planteen otras alternativas más sencillas y baratas En los últimos años se han publicado numerosos trabajos sobre el empleo de técnicas de bajo coste para el análisis de movimientos humanos, especialmente mediante sensores inerciales. Aunque estos sensores pueden proporcionar una buena estimación de los ángulos articulares, no permiten la medida de desplazamientos con suficiente precisión (Picerno, 2017).

Una alternativa es el uso del videoanálisis, que se ha centrado en el desarrollo de sistemas simples para el análisis de movimientos con una sóla cámara. En esta línea se han desarrollado diferentes sistemas sencillos, pero restringidos al análisis de movimientos planos, lo que limita las posibles aplicaciones (Otín et al., 2016).

Sin embargo, en el campo de la robótica se vienen usando desde hace tiempo sistemas de posicionado mediante marcadores de realidad aumentada (AR). Estos sistemas permiten analizar, en tiempo real y con una única cámara de video, la posición y orientación de objetos con precisión suficiente para su uso en muchas aplicaciones biomecánicas (Parrilla et al., 2013). En particular, la librería ArUco (Muñoz-Salinas, 2012) permite analizar movimientos de varios marcadores de manera rápida y eficiente, por lo que se han aplicado al control de robots colaborativos (Maule et al., 2017).

Sin ambargo, a pesar de su potencial, este tipo de marcadores apenas han sido utilizados en el ámbito de la Biomecánica o en aplicaciones clínicas, por lo que no conocemos su precisión y fiabilidad, si bien los pocos estudios publicados muestran las posibilidades de este sistema (Nagymáté and Kiss, 2019).

En esta línea se plantea el presente trabajo, en el que se analiza la precisión de los marcadores 
ArUco en la medida de ángulos y desplazamientos, comparando los movimientos medidos con el sistema con dos técnicas de precisión: encoders de desplazamientos lineales y giros, para las medidas estáticas, y un sistema de videofotogrametría de precisión, para las medidas en movimiento.

\section{Material y métodos}

\subsection{Marcadores ArUco. Instrumentación utilizada}

La librería ArUco fue desarrollada originalmente por Rafael Muñoz y Sergio Garrido en la Universidad de Córdoba. Está basado en OpenCV y permite la detección de varios tipos de etiquetas. Su proceso de detección es mucho más efectivo y rápido que otras librerías, lo que lo hace especialmente adecuado para aplicaciones del análisis de movimientos en tiempo real. Los marcadores ArUco, son marcadores cuadrados compuestos por un borde negro ancho con una matriz binaria interna. El borde negro permite una rápida detección de la imagen y la codificación binaria permite la identficación de marcadores, la aplicación de técnica de detección y corrección de errores (Garrido-Jurado et al, 2014)

El proceso de detección de un marcador se realiza en dos etapas: la detección de candidatos a marcadores y el análisis de codificación. En el proceso de detección, se lleva a cabo una extracción de contornos y un filtrado, junto con el umbral adaptativo, para detectar formas cuadradas. En la segunda etapa, se analiza la codificación interna, mediante la cual se determina si el marcador pertenece a un tipo de etiquetas específico. La posición de las cuatro esquinas del marcador en la imagen y el tamaño real del marcador son suficientes para que el sistema pueda estimar su posición completa en escala absoluta.

La aplicación desarrollada puede detectar hasta 6 marcadores diferentes. Para cada uno de ellos se proporciona como salida el instante de tiempo, la identificación del marcador, la posición del centro del marcador y la orientación del sistema de referencia ligado al marcador, expresada como el vector giro respecto al sistema de referencia de la cámara medido en radianes. Los vectores están expresados en la base del sistema de referencia de la cámara.

La velocidad de adquisición depende de la cámara usada, del ordenador y del número de marcadores. En este trabajo se ha usado una frecuencia de 25 fotogramas por segundo.

\subsection{Aplicación al análisis de movimientos humanos. Dispositivos y análisis cinemático}

Para la captura de movimientos se ha usado una cámara Logitech modelo C920 Pro. La 
cámara se calibra según el procedimiento descrito en el software usando un patrón de cuadrículas.

Para capturar el movimiento no se ha usado un solo marcador por segmento, sino un sistema formado por dos marcadores de $7 \mathrm{~cm}$ de lado que forman un pequeño ángulo (unos $20^{\circ}$ ) (Figura 1). La razón para usar dos marcadores es doble. Por una parte, porque mejora la precisión de las medidas, al usar información redundante. El efecto sobre el error de desplazamientos lineales es una mejora por un factor $\sqrt{ } 2$. La mejora sobre el error en los ángulos depende de la separación entre marcadores y es superior a ese factor (Page, et al, 2009). Por otra, se evita una discontinuidad en el registro de la orientación que aparece cuando un marcador queda paralelo al plano de la cámara o casi paralelo; en este caso hay una indeterminación en la orientación, presentándose discontinuidades en el ángulo y con un aumento notable de los errores. Este problema se evita con dos marcadores que no sean paralelos, ya que siempre habrá al menos un marcador no paralelo a la cámara.

La información sobre la posición y orientación del sistema de marcadores se realiza asignando a cada marcador 4 puntos virtuales, correspondientes al origen del sistema local del marcador y a puntos situados sobre los ejes a una distancia de $10 \mathrm{~cm}$ del origen. Esto equivale a disponer de 8 marcadores técnicos por cada segmento. El análisis cinemático se realiza entonces con los algoritmos descritos en Page et al. (2009), obteniéndose el vector de Rodrigues y el desplazamiento del sistema respecto de una posición inicial de referencia.

\subsection{Validación del sistema de medida}

Para validar el sistema de medida se han realizado dos experimentos, destinados a establecer la validez en la medida de desplazamientos estáticos y en movimiento.

El primer experimento tenía como objetivo determinar el error de medida en desplazamientos estáticos, y analizar el efecto de parámetros experimentales como la distancia del marcador a la cámara, separación del eje o inclinación. Para ello se han usado los montajes que se muestran en la Figura 1, donde el sistema de dos marcadores se desplaza sobre la guía de un encoder lineal o gira ligado a un ángulo. Los encoders son marca Digital Protactor, con una resolución de $0.01 \mathrm{~mm}$ y 0.1 , respectivamente. La incertidumbre de los encoders es de 0.1 $\mathrm{mm}$ y $0.3^{\circ}$.

En el caso de los desplazamientos lineales, se han desarrollado dos experimentos, colocando el encoder paralelo al eje $\mathrm{Z}$ de la cámara (profundidad) o perpendicular al mismo. El sistema se ha ido moviendo desde una posición de referencia en intervalos aproximados de $2 \mathrm{~cm}$ y se han contrastado las diferenciss en el desplazamiento medidas con el encoder y las proporciandas por los marcadores ArUco. Se han realizado repeticiones a diferentes distancias a la cámara $(60 \mathrm{~cm}, 75 \mathrm{~cm}, 100 \mathrm{~cm})$. 


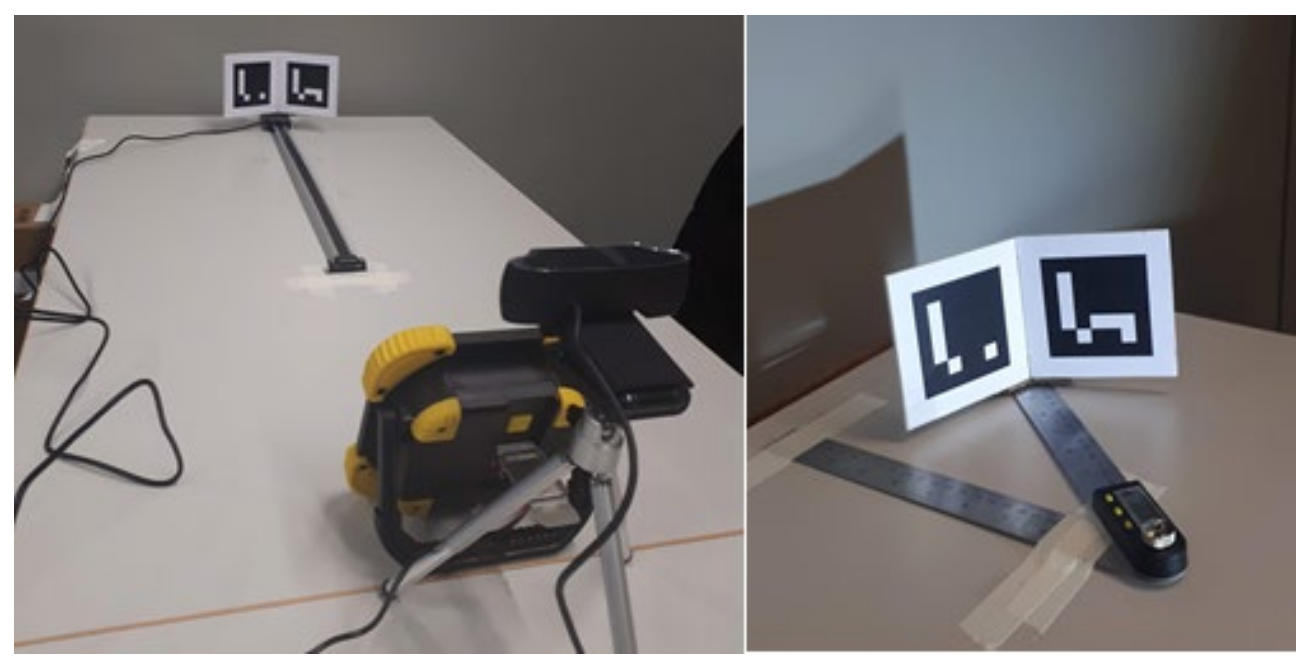

Fig. 1. Montajes para el análisis de los errores en los desplazamientos lineales y angulares estáticos

En el caso de los desplazamientos angulares, se fijó el sistema de marcadores a uno de los brazos del enconder angular y se midieron desplazamientos angulares de hasta $50^{\circ}$ en dos situaciones: con el eje de giro paralelo al eje de la cámara y con el eje de giro del encoder paralelo al plano de la cámara.

En ambos casos se compararon las medidas del sistema ArUco y de los encoders y se obtuvieron dos parámetros de concordancia: el coeficiente de correlación intraclase (ICC) y el error estándar de las medidas (SEM). El ICC se calculó tal como de describe en Weir (2005), usando el tipo ICC(3), al ser el factor "método de medida" un factor fijo. En cuanto al SEM, se ha calculado como la raíz cuadrada del error cuadrático medio en $\mathrm{n}$ anova de dos vías (Weir, 2005).

En el segundo experimento se han comparado las medidas angulares en movimiento, usando una tabla con una bisagra cuyo eje se coloca paralelo al plano de la cámara o perpendicular. Sobre la tabla se fija un sistema de dos marcadores ArUco y un conjunto de marcadores reflectantes. La tabla se movió en un rango aproximado de $50^{\circ}$, a una velocidad normal en análisis de movimientos humanos $(0.5 \mathrm{~Hz})$. El movimiento de la tabla se registró usando los marcadores ArUco y registrando el movimiento con un sistema de videofotogrametría Kinescan-IBV, calibrado para un precisión de $0.3 \mathrm{~mm}$ en la medida de posición y de $0.2^{\circ}$ en la medida de ángulos (Figura 2.).

Al igual que en el caso anterior, la concordancia en cada movimiento se describió mediante el ICC y el SEM. 


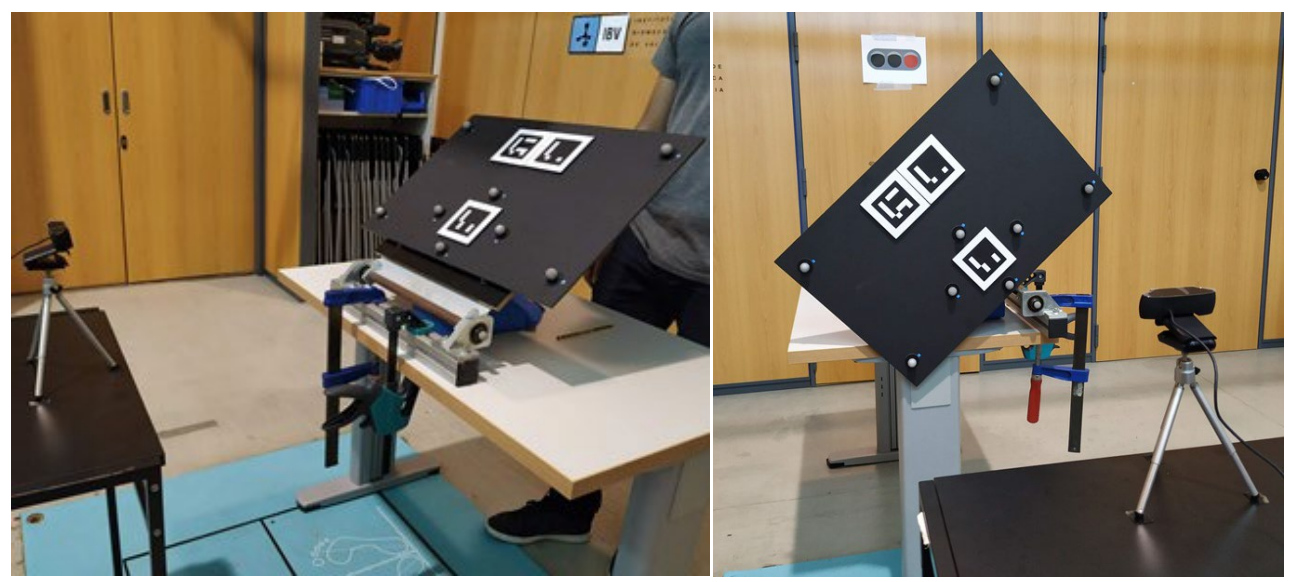

Fig. 2. Comparación entre el movimiento registrado por un sistema e fotogrametría y los marcadores ArUco. El movimiento del sistema ArUco se registra exclusivamente con los dos marcadores en la parte superior de la tabla. El marcador inferior se usa para definir un sistema de referencia común a la cámara de vídeo y al sistema de videofotogrametría.

\section{Resultados y Discusión}

La tabla 1 muestra los valores del ICC entre las medidas con encoders y ArUco, así como el SEM obtenido, para los desplazamientos lineales y angulares estáticos.

Como puede observarse, la medida de desplazamientos estáticos con ArUco es muy precisa. Los ICC tienen valores superiores a 0.999 en todos los casos, lo que muestra una concordancia excelente.

El error asociado a los giros con el eje perpendicular a la cámara es del orden de la precisión del encoder $\left(\mathrm{SEM}=0.2^{\circ}\right)$, mientras que los giros en un eje paralelo a la cámara tienen un error algo mayor ( $\mathrm{SEM}=0.8^{\circ}$ ). Dicha diferencia se debe a que en el segundo caso hay un desplazamiento en profundidad, que se mide con menos precisión con una sóla cámara. Esto indica que en caso de movimientos humanos, la cámara debería colocarse paralela al plano principal del movimiento (por ejemplo, plano sagital en el caso de marcha humana). En cualquier caso, se trata de un error de medida mucho más pequeño que el de otros dispositivos usados en el ámbito clínico, como los inclinómetros y goniómetros que tienen errores del orden de $3^{\circ}$ (Audette et al, 2010). Por otra parte, la variabilidad humana también es muy superior a estos valores. Por ejemplo, en el estudio del rango de flexo-extensión del cuello, la variabilidad de los sujetos al repetir la prueba tiene un SEM del orden de 5.5 (Cagnie et al., 2007). Por tanto, el efecto del pequeño error de medida de este sistema es despreciable frente a las diferencias de comportamiento del mismo sujeto en pruebas diferentes. 
Tabla 1. Concordancia entre las medidas de desplazamientos angulares y lineales estáticos, medido con encoder y con marcadores ArUco

\begin{tabular}{|c|c|c|c|}
\hline & & $\begin{array}{l}\text { Coeficiente de } \\
\text { correlación intraclase } \\
\text { (ICC). Adimensional }\end{array}$ & $\begin{array}{c}\text { Error estandar de la } \\
\text { medida (Giros en }{ }^{\circ} ; \\
\text { desplazamientos en } \mathbf{m m} \text { ) }\end{array}$ \\
\hline \multirow[t]{2}{*}{ Giro } & $\begin{array}{l}\text { Eje paralelo a la } \\
\text { cámara }\end{array}$ & 0,9996 & 0,80 \\
\hline & $\begin{array}{c}\text { Eje perpendicular } \\
\text { a la cámara }\end{array}$ & 1,0000 & 0,20 \\
\hline \multirow[t]{5}{*}{ Desplazamiento } & $\begin{array}{l}\text { Paralelo cámara, } \\
Z=100 \mathrm{~cm}\end{array}$ & 0,9998 & 1,50 \\
\hline & $\begin{array}{l}\text { Paralelo cámara, } \\
\qquad \mathrm{Z}=75 \mathrm{~cm}\end{array}$ & 0,9999 & 1.00 \\
\hline & $\begin{array}{l}\text { Paralelo cámara, } \\
\qquad \mathrm{Z}=60 \mathrm{~cm}\end{array}$ & 0,9999 & 1,20 \\
\hline & $\begin{array}{l}\text { Paralelo al eje de } \\
\text { la cámara, } \\
\text { desplazado } \\
\text { lateralmente }\end{array}$ & 0,9993 & 3,40 \\
\hline & $\begin{array}{l}\text { Paralelo al eje de } \\
\text { la cámara, } \\
\text { centrado }\end{array}$ & 0,9999 & 1.10 \\
\hline
\end{tabular}

En cuanto a los desplazamientos lineales, el error más grande aparece cuando los desplazamientos son en profundidad (eje $\mathrm{Z}$ de la cámara) y además están separados de dicho eje. En este caso los errores son del orden de $3 \mathrm{~mm}$. Por el contrario, el hecho de centrar el eje mejora notablemente la concordancia, obteniéndose un error el orden de $1 \mathrm{~mm}$. Estos resultados indican la conveniencia de centrar bien el campo de medida, de manera que los marcadores aparezcan en la zona central de la imagen.

En el caso de los desplazamientos paralelos al plano de la cámara, el error depende de la separación a la cámara, lo que resulta previsible, dado que al separar los marcadores aparecen más pequeños en la imagen y aumenta el error aleatorio (Page et al., 2008). Para el tamaño de marcador de este estudio, las medidas tienen errores del orden de $1 \mathrm{~mm}$ hasta una distancia de $0.75 \mathrm{~m}$. A distancias mayores, el error crece. No obstante, para las distancias focales que se manejan en las cámaras web esa distancia es suficiente para muchas aplicaciones, por ejemplo para analizar el miembro inferior o el movimiento de la cabeza. Por ello, y al igual que sucede con cualquier sistema de visión por computador, la precisión mejora si se ajusta 
el campo de visión a la zona de movimiento real.

Tabla 2. Concordancia entre las medidas de desplazamientos angulares dinámicos, medido con videofotogrametría y con marcadores ArUco

Coeficiente de correlación intraclase

(ICC). Adimensional

\author{
Error estandar de la \\ medida $\left({ }^{\circ}\right)$
}

\begin{tabular}{ccc}
\hline Giro & $\begin{array}{c}\text { Paralelo al eje de } \\
\text { la cámara }\end{array}$ & 0,9970 \\
$\begin{array}{c}\text { Paralelo a la } \\
\text { cámara }\end{array}$ & 0.9960 & 1.70 \\
& & 1.20 \\
\hline
\end{tabular}

En cuanto a la precisión en la medida de ángulos en movimiento, los resultados aparecen en la Tabla 2. Aunque los ICC siguen siendo excelentes, el error angular es superior a $1^{\circ}$ tanto en los giros respecto de un eje paralelo al eje de la cámara $\left(\mathrm{SEM}=1.20^{\circ}\right)$, como en un eje paralelo a la cámara $\left(\mathrm{SEM}=1.7^{\circ}\right)$. Estos resultados presentan errores mayores que los obtenidos por Parrilla et al (2013), si bien las diferencias pueden deberse al tipo de movimiento analizado en dicho trabajo, que estaba confinado en un espacio más pequeño, o a los algoritmos de cálculo que en nuestro estudio se realizan en tiempo real. Por el contrario, los errores obtenidos en nuestro trabajo son bastante menores que los descritos por Nagymate et al. (2019) en un estudio sobre marcha. En nuestra opinión, los pobres resultados mostrados en dicho estudio se deben más a la presencia de artefactos por movimiento de tejidos blandos que a la precisión del equipo.

En cualquier caso se trata de errores angulares muy pequeños y asumibles en muchas aplicaciones, como la medida de rangos de movimiento de la cabeza o brazo. Como se ha indicado anteriormente, la variabilidad intrasujeto es mayor, en muchos casos a este nivel de errores.

\section{Conclusiones}

A la vista de los resultados obtenidos en este trabajo, cabe concluir que los marcadores ArUco son una herramienta de gran potencial en el desarrollo de sistemas de análisis de movimientos para aplicaciones clínicas. No sólo permite hacer mediciones en tiempo real y con un coste muy bajo (una webcam de calidad cuesta menos de 100 euros), sino que ofrece niveles de precisión suficientes. Así, los errores obtenidos son bastante menores que los asociados a la 
variabilidad humana y menores que los de otros equipos como inclinómetros. Es un sistema libre de derivas y sin problemas a la hora de interpretar la posición de los sistemas de referencia

Sin embargo, la calidad de las medidas depende del diseño del sistema experimental, que debe verificar algunas condiciones, como estar centrado en el centro de la imagen y con un campo bien ajustado a la amplitud del movimiento.

\section{Agradecimientos}

Este trabajo ha sido financiado por el Gobierno de España y cofinanciado por Fondos Feder de la UE gracias al proyecto PDI2017-84201-R (IMBIO3R).

\section{Referencias}

AUDETTE, I., DUMAS, J. P., COTE, J. N., \& DE SERRES, S. J. (2010). « Validity and between-day reliability of the cervical range of motion (CROM) device». Journal of Orthopaedic \& Sports PhysicalTtherapy, vol. 40, issue 5, pp. 318-323.

CAGNIE, B., COOLS, A., DE LOOSE, V., CAMBIER, D., \& DANNEELS, L. (2007). « Reliability and normative database of the Zebris cervical range-of-motion system in healthy controls with preliminary validation in a group of patients with neck pain». Journal of Manipulative and Physiological Therapeutics, vol. 30, issue 6, pp. 450-455.

GARRIDO-JURADO, S., MUÑOZ-SALINAS, R., MADRID-CUEVAS, F. J., \& MARIN-JIMENEZ, M. J. (2014). «Automatic generation and detection of highly reliable fiducial markers under occlusion », Pattern Recognition, vol. 4, issue 6, p,2280-2292.

MAULE, L., FORNASER, A., TOMASIN, P., TAVERNINI, M., MINOTTO, G., DA LIO, M., \& DE CECCO, M. (2017). « Augmented robotics for electronic wheelchair to enhance mobility in domestic environment ». In : International Conference on Augmented Reality, Virtual Reality and Computer Graphics (pp. 22-32). Springer, Cham.

MUÑOZ-SALINAS, R. (2012). «Aruco: a minimal library for augmented reality applications based on OpenCV ». Universidad de Córdoba.

NAGYMATE, G., \& KISS, R. M. (2019). «Affordable gait analysis using augmented reality markers ». PloS one, vol. 14, issue 2, e0212319.

OTIN, C. (2016). «Análisis de habilidades deportivas mediante el uso del software Kinovea ». In Simbiosis del aprendizaje con las tecnologías: experiencias innovadoras en el ámbito hispano (pp. 125134). Prensas Universitarias de Zaragoza.

PAGE, Á., DE ROSARIO, H., MATA, V., \& ATIENZA, C. (2009). « Experimental analysis of rigid body motion. A vector method to determine finite and infinitesimal displacements from point coordinates ». Journal of Mechanical Design, vol. 131, issue 3, pp. 031005. 
PAGE, A., MORENO, R., CANDELAS, P., \& BELMAR, F. (2008). «The accuracy of webcams in 2D motion analysis: sources of error and their control ». European Journal of Physics, vol. 29, issue 4, pp. 857.

PARRILLA, E., et al.. (2013). « Ankle 3D-kinematics measurement by using a single camera and ARmarkers ». Footwear Science, vol. 5, sup.1, pp. 73-74.

PICERNO, P. (2017). "25 years of lower limb joint kinematics by using inertial and magnetic sensors: a review of methodological approaches“. Gait \& Posture, vol. 51, p. 239-246.

WEIR, J. P. (2005). «Quantifying test-retest reliability using the intraclass correlation coefficient and the SEM ». The Journal of Strength \& Conditioning Research, 19(1), 231-240. 